\title{
Nailfold videocapillaroscopy micro-haemorrhage and giant capillary counting as an accurate approach for a steady state definition of disease activity in systemic sclerosis
}

\author{
Domenico Sambataro ${ }^{1 *}$, Gianluca Sambataro', Eleonora Zaccara', Wanda Maglione', Riccardo Polosa²,
} Antonella MV Afeltra ${ }^{3}$, Claudio Vitali ${ }^{4}$ and Nicoletta Del Papa ${ }^{1}$

\begin{abstract}
Introduction: Nailfold videocapillaroscopy (NVC) in systemic sclerosis (SSC) is a procedure commonly used for patient classification and subsetting, but not to define disease activity (DA). This study aimed to evaluate whether the number of micro-haemorrhages (MHE), micro-thrombosis (MT), giant capillaries (GC), and normal/dilated capillaries (Cs) in NVC could predict DA in SSC.
\end{abstract}

Methods: Eight-finger NVC was performed in 107 patients with SSC, and the total number of MHE/MT, GC, and the mean number of Cs were counted and defined as number of micro-haemorrhages (NEMO), GC and Cs scores, respectively. The European Scleroderma Study Group (ESSG) index constituted the gold standard for DA assessment, and scores $\geq 3.5$ and $=3$ were considered indicative of high and moderate activity, respectively.

Results: NEMO and GC scores were positively correlated with ESSG index $(R=0.65, P<0.0001$, and $R=0.47, P<0.0001$, respectively), whilst $C s$ score showed a negative correlation with that $D A$ index $(R=-0.30, P<0.001)$. The area under the curve (AUC) of receiver operating characteristic plots, obtained by NEMO score sensitivity and specificity values in classifying patients with ESSG index $\geq 3.5$, was significantly higher than the corresponding AUC derived from either GC or Cs scores $(P<0.03$ and $P<0.0006$, respectively). A modified score, defined by the presence of a given number of MHE/MT and GC, had a good performance in classifying active patients (ESSG index $\geq 3$, sensitivity $95.1 \%$, specificity $84.8 \%$, accuracy $88.7 \%)$.

Conclusions: MHE/MT and GC appear to be good indicators of DA in SSc, and enhances the role of NVC as an easy technique to identify active patients.

\section{Introduction}

Systemic sclerosis ( $\mathrm{SSc}$ ) is a connective tissue disease clinically characterized by Raynaud's phenomenon, and progressive fibrotic changes in the skin and internal organs, such as heart, lung, and kidney. Subsets of SSc have been defined, that is, limited cutaneous (lc) SSc, diffuse cutaneous (dc) SSc and SSc without skin involvement [1].

As in other systemic autoimmune diseases, SSc has a clinical course characterized usually by early phases of

\footnotetext{
* Correspondence: d.sambataro@hotmail.it

'U.O.C. Day Hospital Reumatologia, Ospedale Gaetano Pini, Via Gaetano Pini, 9-20122 Milano, Italy

Full list of author information is available at the end of the article
}

activity. A small vessel vasculopathy, characterised by peripheral vascular and interstitial mononuclear cell infiltration, represents the hallmark of the disease in the early phase. Conversely, in the late disease stages, the entire process may evolve to irreversible fibrotic changes of the involved tissues and organs [2-4]. This should induce the clinician to define an early diagnosis and recognize any active phase of the disease, in order to contrast the irreversible final damage by introducing an appropriate treatment [5-7].

The assessment of disease activity (DA) in SSc is a rather difficult challenge, and only a composite index that 
includes several clinical, instrumental, and serological items has, so far, been proposed and validated $[8,9]$.

Nailfold videocapillaroscopy (NVC) is a simple method that allows the clinician to observe and follow up the disease-related micro-vascular changes in an easily accessible capillary bed [10]. NVC plays an important role in the diagnosis of SSc and is included as an item even in the 2013 classification criteria for SSc [11]. In addition, different NVC patterns have been defined and their prognostic value underlined [12-14]. Finally, NVC has been used to define the different phases of the disease. This allows distinguishing patients with early, active, and late NVC pattern, where the active pattern is defined by the presence of numerous ectasic and giant capillaries (GC), ramified capillaries, micro-haemorrhages (MHE) and micro-thrombosis (MT), and initial loss of capillaries with avascular areas [15].

In the present study, we reconsider the specific NVC abnormalities that have been suggested to be increased (MHE, MT, and GC), or progressively reduced (number of capillaries, Cs) during the active phases of disease, to verify whether some quantitative combination of these features could be useful to identify patients with a relevant level of DA.

We decided to assess the presence of MT together with that of MHE separately from that of GC.

This statement was based on the present interpretation of micro-vascular NVC abnormalities in SSc. It has been suggested that MT and MHE are strictly related to each other, and represent the final evolution of enlarged capillary loops in a rapidly progressive pathological process [16]. Conversely, GC formation is certainly the first response to micro-vascular aggression and damage [17], but they may remain substantially unchanged when repeatedly observed in NVC in a slowly evolving or stable disease [18]. Consequently, the progressive reduction of the numbers of Cs can be regarded as the final result of capillary bed pathological aggression, and also of the substantial failure of capillary reconstruction attempts $[17,18]$.

\section{Methods}

\section{Patients}

One hundred and seven patients who met the American College of Rheumatology/European League Against Rheumatism (ACR/EULAR) classification criteria for SSc [11] formed the study population. They were also classified as having lcSSc or dcSSc following the LeRoy criteria [1]. Current pregnancy, diabetes, smoking and onicophagic habitus, presence of anti-phospholipid antibodies, and assumption of beta blockers, all conditions that may influence the NVC pattern, were considered as exclusion criteria from the study. At the moment of study enrolment, 49 out of the 107 patients were treated with infusion of intravenous prostanoids ( 42 with monthly iloprost and 7 with weekly alprostadil), and 8 were taking bosentan. Furthermore, all of the enrolled patients were under treatment with low-dose acetylsalicylic acid.

\section{Nailfold videocapillaroscopy}

The nailfold capillaries of all fingers of both hands, excluding thumbs, were examined in each patient using a videocapillaroscope with a $200 \times$ magnification lens. Four consecutive 1-mm fields for a total extension of $4 \mathrm{~mm}$ in the middle of nailfold were examined. The derived digital images were then stored and analysed by using dedicated software (Videocap Scalar Co. Ltd, DS MediGroup, Milan, Italy).

In Figure 1 some typical examples of what we have considered as capillaroscopic MHE and MT are shown [19].

The cumulative number of MHE and MT observed in the images obtained from eight fingers in each patient was calculated, and defined as the NEMO score (number of micro-haemorrhages). Each separate MHE and MT was counted as one in this score, independently of its size. Similarly, the total number of GC, defined as capillaries with a diameter over $50 \mu \mathrm{m}$ [17], and the mean number of normal or slightly dilated Cs observed in the same NVC fields, were also counted in each patient, and defined as the GC and Cs scores, respectively.

The NVC images were used to subclassify patients in different disease stages, according to a previously proposed method [15] that indicated three different capillaroscopic patterns: (i) 'early pattern', classified as initial stage of disease, characterized by the presence of few GC and capillary MHE, no evident loss of Cs and relatively well-preserved capillary distribution; (ii) 'active pattern', classified as the acclaimed stage of disease, characterized by numerous GC and capillary MHE, moderate capillary loss and mildly disorganized capillary architecture; and (iii) 'late pattern', as advanced disease, with few or absence of GC and MHE, extensive capillary loss, and severe disorganization of the normal capillary architecture.

\section{Clinical work-up and assessment of disease activity}

The European Scleroderma Study Group (ESSG) index was taken as the gold standard for DA assessment $[8,9]$. This a composite scoring system that includes a number of clinical, instrumental, and serological items, derived by a multivariate analysis performed in a cohort of patients with both lcSSc and dcSSc [8]. A second study carried out in a completely different cohort of patients allowed to finally demonstrate that this scale was a reliable and a valid method to assess DA in SSc [9]. Following the ESSG indications, a score $\geq 3.5$ was considered to have a good sensitivity and specificity in separating patients with high DA level. In addition, we also took into account a cut-off value of 3.0 [20] in order to also include patients with moderate level of DA. A clinical work- 

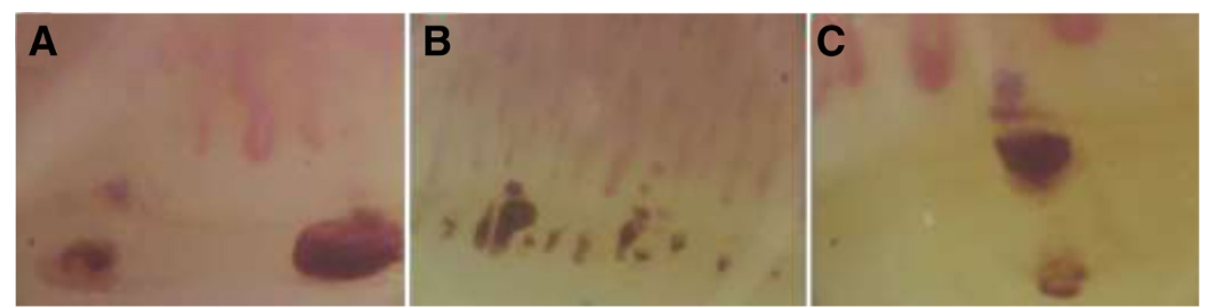

Figure 1 Example of haemosiderin deposits taken into account when calculating the NEMO score. (A) Two micro-haemorrhages of relevant size are evident in the cuticle border, as a result of erythrocyte extravasation following the rupture of capillaries. The consequent haemosiderin deposits assume a round form; (B) numerous synchronous micro-haemorrhages are observed aligned in the distal row of the cuticle. Synchronicity of events is defined by the presence of haemosiderin deposits in the distal row of capillaries aligned at the same level [16]; (C) a haemosiderin deposit that mirrors the shape of a capillary loop is shown. This aspect is considered indicative of a micro-thrombosis [19]. NEMO, number of micro-haemorrhages.

up needed for ESSG score definition was completed within the month of NVC performance.

The modified Rodnan skin sScore (mRSS), that is, an important item also included in the ESSG activity index, was performed by experienced physicians (NDP, WM, EZ) other than those who performed NVC (DS, GS). This score is the most widely used method to assess the skin thickening by the exploration of 17 different body sites. The clinician scores skin thickness on a scale ranging from 0 to 3 . The mRSS has showed to be a valid method to assess skin involvement in SSc, closely related to the skin histological features, and to have a good inter-observer reliability [21-23].

\section{Statistical analysis}

Statistical analysis was performed by standard procedures using IBM SPSS ${ }^{\text {Tx }}$ Statistics 21 (IBM, Armonk, NY, USA) and GraphPad Prism ${ }^{\text {mo }} 6$ software package (GraphPad Software Inc., La Jolla, CA, USA).

Since NEMO, GC and Cs scores did not appear to have a normal distribution, we used non-parametric tests to compare these variables with other categorical or ordinal variables taken into account in the study.

A logistic regression model was also tested to assess the contribution of defined values of NEMO, GC and Cs scores in predicting the presence of different level of DA according to predefined ESSG cut-off values.

Receiver operating characteristic (ROC) curves were constructed by plotting sensitivity and specificity values of NEMO, GC and Cs scores in correctly classifying patients having or not having an active disease phase. The HanleyMcNeil test was applied to test the significance of the differences between the areas under the ROC curves obtained from the analysed NVC variables.

Weighted Cohen's k statistics were preliminarily applied to evaluate the interobserver agreement in both counting NVC abnormalities and assessing mRSS, and resulted to be good in both cases ( 0.69 and 0.75 , respectively).
No correction of the statistical results was made for the presence of missing values, since no data were missed in our database.

\section{Ethical rules}

This study was conducted according to the Helsinki Declaration and approved by the ethics committee of 'Ospedale G. Pini, Milan, Italy', where the study was carried out and all the study patients were recruited. A written informed consent was obtained from all of the enrolled patients.

\section{Results}

The patients with SSc had a mean age of 55.8 yrs. (range 18 to $84 \mathrm{yrs}$.) and mean disease duration of $6.1 \mathrm{yrs}$. (range 0 to 30 yrs.). As expected, females represented the large majority of the study population (97/107). The main demographic, clinical and laboratory data of the patients are summarised in Table 1.

In accordance with the ESSG index pre-defined cut-off value suggested to distinguish patients with high level of DA, that is, an ESSG index $\geq 3.5$, 32 out of the 107 patients could be considered as very active (14 with dcSSc and 18 with lcSSc). Conversely, active patients became 41 when an ESSG index $\geq 3.0$ was used as cut-off value to also included patients with moderate DA (26 with lcSSc and 15 with dcSSc). The main clinical characteristics of nine additional active patients were reported in Table 2. It appears evident that all of these patients showed relevant aspects suggesting a phase of DA.

The NEMO score appeared to be a good predictor for DA since NEMO values were strictly correlated with both ESSG index scores (Spearman's $\mathrm{R}=0.65, P<0.0001$ ), and with $\mathrm{mRSS}(\mathrm{R}=0.59, P<0.001)$, but not with patients' age and disease duration. When the other components of ESSG activity index were considered, NEMO score appeared to be significantly higher in patients presenting with scleredema (Mann-Whitney $U \mathrm{Z}$ value $=4.59$, $P<0.0001)$, worsening of skin $(\mathrm{Z}=5.79, P<0.0001)$, 
Table 1 Demographic, clinical, and serological features, according to ESSG index $[8,9]$

\begin{tabular}{|c|c|c|c|}
\hline & Whole cohort & Patients with IcSSc & Patients with dcSSc \\
\hline Patients (F/M) & $107(97 \mathrm{~F}, 10 \mathrm{M})$ & $57(53 \mathrm{~F}, 4 \mathrm{M})$ & $50(44 \mathrm{~F}, 6 \mathrm{M})$ \\
\hline Mean age (range) (yrs.) ${ }^{1}$ & $55.8(18-84)$ & $58.1(28-84)$ & $53.3(18-84)$ \\
\hline Mean disease duration (range) (yrs.) & $6.1(0.5-30)$ & $7.4(0.5-30)$ & $4.8(0.5-20)$ \\
\hline ESSG index $\geq 3.5$ (no. of pts. $)^{2}(\%)$ & $32(29.9 \%)$ & $18(16.8 \%)$ & $14(13.1 \%)$ \\
\hline Mean mRSS (range) (yrs.) & $3.1(0-32)$ & $2.4(0-5)$ & $3.5(0-32)$ \\
\hline Scleredema (no. of pts.) (\%) & $60(56.1 \%)$ & $32(56.1 \%)$ & $28(56.0 \%)$ \\
\hline$\Delta^{3}$ Skin involvement (no. of pts.) (\%) & $18(16.8 \%)$ & $11(19.2 \%)$ & $7(14.0 \%)$ \\
\hline Ulcers (no. of pts.) (\%) & $22(21.5 \%)$ & $11(19.2 \%)$ & $11(22.0 \%)$ \\
\hline$\Delta^{1}$ Vascular features (no. of pts.) (\%) & $25(23.4 \%)$ & $14(24.5 \%)$ & $11(22 \%)$ \\
\hline Arthritis (no. of pts.) (\%) & $5(4.7 \%)$ & $3(5.2 \%)$ & $2(4 \%)$ \\
\hline DLCO $<80 \%{ }^{4}$ (no. of pts.) (\%) & $67(63.2 \%)$ & $35(61.4 \%)$ & $32(64.0 \%)$ \\
\hline$\Delta^{1}$ Cardiopulmonary features (no. of pts.) (\%) & $20(18.7 \%)$ & $10(17.5 \%)$ & $10(20.0 \%)$ \\
\hline ESR >30 mm/h (no. of pts.) (\%) & $32(29.9 \%)$ & $14(24.5 \%)$ & $18(36.0 \%)$ \\
\hline Hypocomplementemia (no. of pts.) (\%) & $12(11.7 \%)$ & 9 (15.7\%) & $3(6.0 \%)$ \\
\hline
\end{tabular}

${ }^{1}$ Yrs. = years; ${ }^{2}$ pts = patients; ${ }^{3}$ worsening in the specific organ/system involvement; ${ }^{4}$ a diffusing lung capacity for carbon monoxide below $80 \%$ of predicted value. ESSG, European Scleroderma Study Group; IcSSc, limited cutaneous systemic sclerosis; dcSSc, diffuse cutaneous systemic sclerosis; mRSS, modified Rodnan skin score; DLCO, diffusing capacity; ESR, erythrocyte sedimentation rate.

cardio-pulmonary $(Z=3.59, P<0.0005)$, and vascular features $(Z=3.18, P<0.002)$, current digital ulcers $(Z=2.91$, $P<0.005)$, and erythrocyte sedimentation rate (ESR) over $30 \mathrm{~mm} / \mathrm{h}(\mathrm{Z}=3.79, P<0.0001)$.

Similarly, also the GC score was significantly correlated with ESSG index score and with mRSS $(\mathrm{R}=0.47$, $P<0.0001$, and $\mathrm{R}=0.34, P<0.001$, respectively). Among the different components of ESSG scoring system, GC score was significantly associated with the presence of scleredema $(Z=4.8, P<0.0001)$, digital ulcers $(Z=2.80$,
$P<0.01)$ and worsening of cutaneous $(\mathrm{Z}=3.72$, $P<0.0005)$, vascular $(Z=2.64, P<0.01)$, and cardiopulmonary $(Z=2.12, P<0.05)$ features.

As expected, negative correlations were found between Cs score and both ESSG index and mRSS $(\mathrm{R}=-0.27$, $P=0.004$, and $\mathrm{R}=-0.26, P=0.007)$. Furthermore, $\mathrm{Cs}$ score was significantly lower in patients with scleredema $(\mathrm{Z}=-3.02, P=0.003)$, digital ulcers $(\mathrm{Z}=-3.08, P=0.002)$, and diffusing capacity (DLCO) $<80 \%$ of predicted value $(\mathrm{Z}=-3.4, P=0.001)$.

Table 2 Disease activity assessment of nine patients with ESSG score $=3$

\begin{tabular}{|c|c|c|c|c|c|c|c|c|c|}
\hline Patient code & 15 & 37 & 43 & 53 & 65 & 69 & 89 & 90 & 93 \\
\hline mRSS & 0 & 2 & 4 & 5 & 8 & 4 & 6 & 6 & 0 \\
\hline Scleredema & - & + & + & + & + & + & + & + & + \\
\hline$\Delta^{1}$ Skin features & - & - & - & - & + & - & - & - & - \\
\hline Ulcers & - & - & + & - & - & - & - & + & + \\
\hline$\Delta^{1}$ Vascular features & - & - & - & - & + & + & + & - & + \\
\hline Arthritis & + & - & - & - & - & - & + & - & - \\
\hline DLCO $<80 \%{ }^{2}$ & + & + & + & + & - & + & - & + & - \\
\hline$\Delta^{1}$ Cardiopulmonary features & + & + & - & + & - & - & - & - & - \\
\hline $\mathrm{ESR}>30 \mathrm{~mm} / \mathrm{h}^{3}$ & + & + & - & + & - & - & - & - & - \\
\hline Hypocomplementemia & - & - & - & - & - & - & - & - & - \\
\hline NVC pattern & Active & Active & Active & Early & Early & Active & Active & Active & Active \\
\hline NEMO score & 7 & 12 & 3 & 0 & 3 & 6 & 19 & 9 & 39 \\
\hline GC score & 9 & 3 & 7 & 2 & 5 & 3 & 10 & 11 & 5 \\
\hline Cs score & 7 & 8 & 7 & 9 & 9 & 5 & 8 & 7 & 7 \\
\hline
\end{tabular}

${ }^{1}$ Worsening in the specific involvement; ${ }^{2}$ a diffusing lung capacity for carbon monoxide below $80 \%$ of predicted value; ${ }^{3}$ an erythrocyte sedimentation rate over $30 \mathrm{~mm} / \mathrm{h}$. ESSG, European Scleroderma Study Group; mRSS, modified Rodnan skin score; DLCO, diffusing capacity; ESR, erythrocyte sedimentation rate; NVC, nailfold videocapillaroscopy; NEMO, number of micro-haemorrhages; GC, giant capillaries; Cs, capillaries. 
The ROC curves constructed by plotting the sensitivity and specificity values of different NEMO, GC and Cs scores in correctly classifying active patients defined by ESSG index cut-off values of both 3.5 and 3.0 were represented in Figure 2. Generally speaking, NEMO score works better with respect to GC and Cs scores in identifying patients with active disease, and the area under the curve (AUC) of the NEMO score ROC plots were higher than the corresponding AUC of either GC or Cs ROC curves. Both differences reached the level of significance $(P<0.03$ and $P<0.0006$, respectively, considering the twotailed Hanley-McNeil test) when the ESSG index cut-off value of 3.5 was taken into account. Similarly the AUC of the NEMO score was significantly higher than that of the GC score and Cs score when the ESSG index cut-off value of 3.0 was considered $(P<0.05$ and $P<0.0001$, respectively; see Figure 2 for details).

A NEMO score $\geq 6$, a GC score $\geq 3$, and a Cs score $\leq 6$ showed the most balanced performance in terms of sensitivity/specificity ratio and the best accuracy in correctly classifying patients with active disease at both cut-off values considered (Table 3).

When the distribution of NEMO score according to the NVC patterns was analysed, it appears evident that the highest NEMO score values and majority of patients with NEMO scores $\geq 6$ were found in the population of patients classified as having the 'active' NVC pattern [15] $(27 / 43,62,8 \%)$. However, a relevant number of patients with a NEMO score $\geq 6$ could also be found in the 'early' and 'late' SSc population [15]. Eight out of eleven patients with the early and all of the five with the late scleroderma pattern having a NEMO score $\geq 6$ had an ESSG index $\geq 3.5$.

When a NEMO score $\geq 6$, a GC score $\geq 3$ and a Cs score $\leq 6$ were analysed all together as predictors of an active phase of disease (ESSG score $\geq 3.0$ ), by building a logistic regression model, only NEMO and GC variables gave a significant contribution to the model, although the odds ratio of NEMO score was much higher with respect to that of GC score (Table 4).

With this figure in mind, we tested sensitivity, specificity and accuracy of a modified ( $\mathrm{m}$ ) NEMO score where a classification of the patients as active was indicated by the presence of $\geq 6 \mathrm{MHE} / \mathrm{MT}$, or - alternatively - by the contemporary presence of $<6 \mathrm{MHE} / \mathrm{MT}$ plus a variable number of GC. A mNEMO defined by the presence of $\geq 6$ $\mathrm{MHE} / \mathrm{MT}$, or alternatively by the presence of three to five MHE/MT plus at least three GC showed the best accuracy $(88.7 \%)$ in correctly classifying patients with either moderate or high active disease (with an ESSG score $\geq 3$ ), with a sensitivity of $95.1 \%$ and a specificity of $84.8 \%$, and an odds ratio of 109.2 (95\% CI 22.7 to $526.1, P<0.0001$ ).

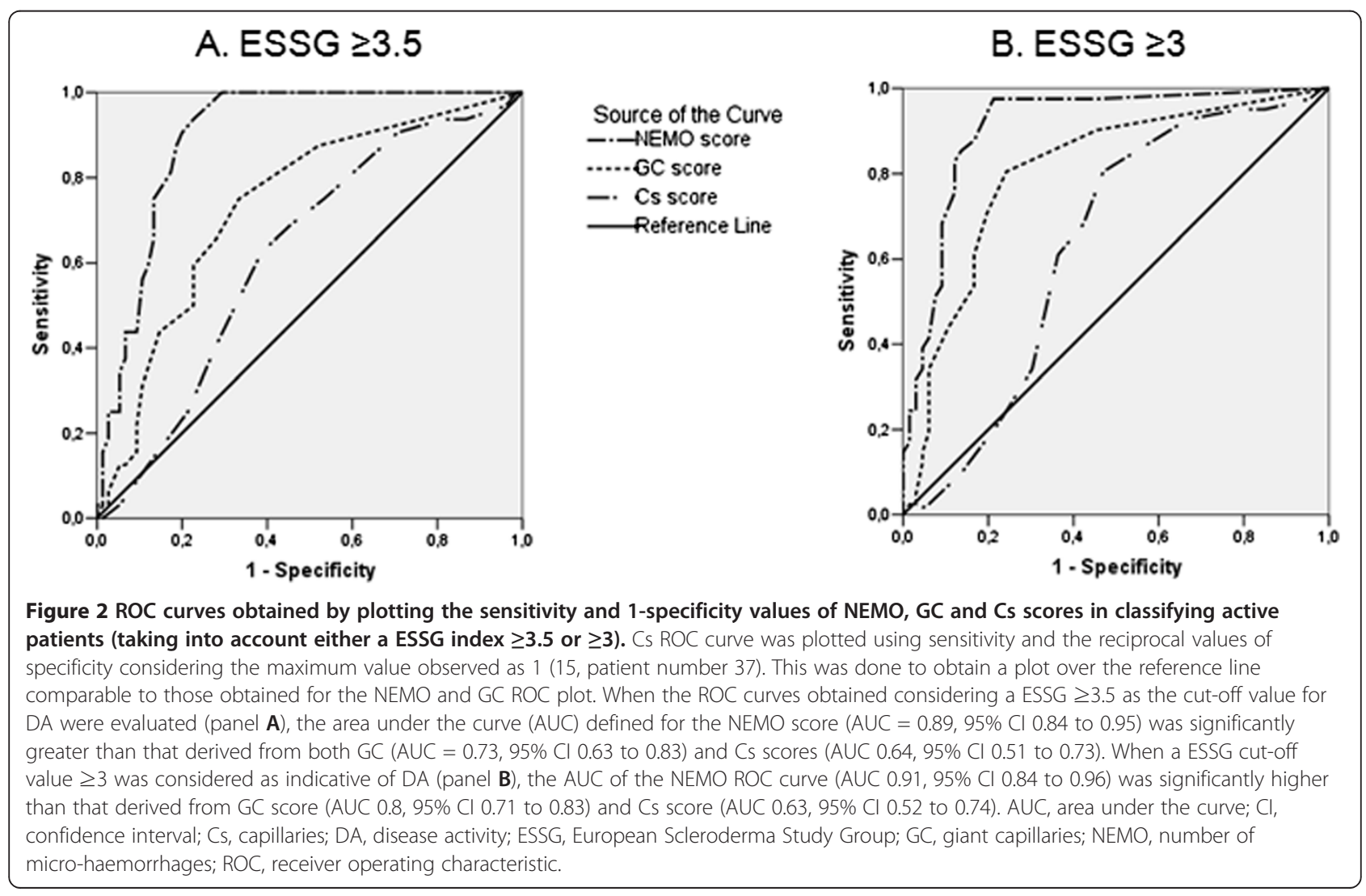


Table 3 Performance of NEMO, GC, and Cs scores in classifying patients with different ESSG scores

\begin{tabular}{lclc}
\hline NEMO score $\geq \mathbf{6}$ for & ESSG $\geq \mathbf{3}$ & \multicolumn{2}{l}{ NEMO score $\geq \mathbf{6}$ for ESSG $\geq \mathbf{3 . 5}$} \\
\hline Sensitivity & 82.9 & Sensitivity & 87.5 \\
Specificity & 87.9 & Specificity & 81.3 \\
Accuracy & 86.0 & Accuracy & 83.2
\end{tabular}

GC score $\geq 3$ for ESSG $\geq 3$

$\begin{array}{lclc}\text { Sensitivity } & 70.7 & \text { Sensitivity } & 65.6 \\ \text { Specificity } & 80.3 & \text { Specificity } & 72.0 \\ \text { Accuracy } & 76.6 & \text { Accuracy } & 70.0 \\ \text { Cs score } \leq \mathbf{6} \text { for ESSG } \geq \mathbf{3} & \text { Cs score } \leq \mathbf{6} \text { for ESSG } \geq \mathbf{3 . 5} \\ \text { Sensitivity } & 40 & \text { Sensitivity } & 40.6 \\ \text { Specificity } & 62.5 & \text { Specificity } & 70.6 \\ \text { Accuracy } & 54.6 & \text { Accuracy } & 61.1\end{array}$

NEMO, number of micro-haemorrhages; GC, giant capillaries; Cs, capillaries; ESSG, European Scleroderma Study Group.

\section{Discussion}

In this study, we demonstrated that the presence of a given number of MHE and MT is highly indicative of an active phase of disease. Even the presence of a sufficient number of $\mathrm{GC}$, and a reduced number of Cs may suggest the presence of DA, although the strength of correlation is higher for the former NVC abnormalities. A score where the presence of a defined number of MHE plus MT, and of a given amount GC were combined derived by a logistic regression model - showed the best predictive value in selecting patients with a current level of moderate and high DA.

NVC is a simple technique largely adopted in clinical assessment of patients with SSc and other connective tissue diseases [24]. Different applications have been described for NVC in SSc. First of all, NVC has an important diagnostic value in SSc, and a specific pattern has been described as characteristic of the disorder, generically defined as belonging to the scleroderma spectrum [17]. Moreover, the recent ACR-EULAR classification criteria for SSc include NVC among the proposed diagnostic items [11]. A relevant prognostic value has also been ascribed to NVC, and different NVC patterns have been defined to be predictive for a worse outcome

Table 4 Multiple logistic regression model evaluating the statistical contribution of given values of different NVC scores as a predictor of DA (ESSG $\geq 3$ )

\begin{tabular}{|c|c|c|c|}
\hline Variables & Odds ratio & $95 \% \mathrm{Cl}^{*}$ & $P$ value \\
\hline NEMO score $\geq 6$ & 22.05 & $6.98-69.64$ & $<0.0001$ \\
\hline GC score $\geq 3$ & 3.62 & $1.14-11.5$ & 0.02 \\
\hline Cs score $\leq 6$ & 2 & $0.57-7.5$ & 0.26 \\
\hline
\end{tabular}

*95\% confidence interval. NVC, nailfold videocapillaroscopy; DA, disease activity; ESSG, European Scleroderma Study Group; NEMO, number of micro-haemorrhages; GC, giant capillaries; Cs, capillaries.
[12-14], and for some specific complications of the disorders $[25,26]$. Finally, NVC has also been used to subclassify patients with SSc according with the phases of disease course, and different NVC patterns described for early, active, and late disease [15]. Conversely, so far, no studies have been carried out specifically aimed at evaluating the potential utility of NVC in stratifying patients according to the level of DA.

The fact that, according to our results, MHE and MT are the NVC abnormalities more strictly indicative of a current DA status is not completely surprising. SSc is usually defined as a disease of the micro-vascular bed of the skin compartment, but potentially compromising the small vessels of internal organs. The endothelial involvement is considered the 'primum movens' of the pathological process in SSc, while migration of mononuclear inflammatory cells in the interstitium, activation of myofibroblast and fibroblast lineage, with the consequent increased production of extracellular matrix and then fibrosis, could be considered as the following stages [2-4]. A large body of evidence indicates that the typical response to the initial micro-vascular damage and capillary loss in SSc is represented by the compensatory dilatation of remaining capillary loops with formation of GC [17]. In slowly evolving or stable disease, it is not unusual to observe GC unchanged over time. On the contrary, in progressive disorder, the destiny of enlarged capillaries is thrombotic obliteration followed by extravasation. When these phenomena are synchronous in many capillaries, serial extravasations with hemosiderin deposits can be observed in the NVC, aligned distally in the cuticle [16].

The finding that the presence of a given amount of MHE and MT (more strongly than that of GC) is associated with DA phases in SSc seems to confirm that these specific abnormalities could represent the NVC counterpart of a rapidly evolution of micro-vascular involvement in active disease phases. The fact that the correlation is less strong for GC can be ascribed to the well-know finding that some GC may appear stable over time in sequential NVC examinations, and then their presence cannot reflect an active phase of the disease. Conversely, the reduction in the number of Cs may be regarded as the final outcome of pathological process in the capillary bed, once the capillary reconstruction attempts have failed. Of course, in a longitudinal study, where a rapidly progressive reduction of capillary number may be assessed in two or more consecutive NVC examinations, one can certainly consider this finding as expression of an active evolving phase of the disease. This is not the case of the present cross-sectional study in which we demonstrated that the current state of DA is more closely represented by the presence of $\mathrm{MH}$ and MT. This statement is certainly reinforced by the observation that MHE and MT, but not GC, have been described in other disorders 
where the involvement of micro-vascular compartment is an important pathological aspect, although induced by partially different mechanisms [27-30].

Since in a specific logistic model the presence of a certain number GC appear to be also predictive for DA, although to a lesser extent, we checked whether a given combination of all these NVC abnormalities could better identify patients with a relevant DA. The modified score (mNEMO score), in which both MHE/MT and GC were included, demonstrated to be actually more accurate and valid for this purpose.

We are, of course, aware that any instrument devoted to assess DA should be not only valid and reliable but also sensitive to change. Not a cross-sectional study like the present one, but a longitudinal study, where the instrument is used to assess changes of DA over time, is certainly needed to verify this latter characteristic.

The here proposed NVC scoring system named mNEMO appears a feasible instrument to assess DA in SSc, since it is possible to predict an active phase of disease during an outpatient visit and in a rather short time. On the contrary, to evaluate the same entity with the previously proposed methods implies a complicate and timeconsuming patient work-up. Of course, patients in whom this specific NVC approach may suggest an active phase of disease should be addressed to a more careful clinical, instrumental and serological evaluation to confirm the suspicion and more precisely define the specific clinical profile. Once a patient has been definitely classified as being in an active phase of disease, a more aggressive therapeutic strategy should be taken into consideration, in accordance with the present recommendations that suggest treating active patients as soon as possible, in order to tentatively modify the natural course of SSc.

\section{Conclusions}

Some specific features observed during NVC examination, such as MHE/MT and GC are strongly predictive of moderate or high level of DA in SSc. A scoring system derived by these findings has been developed and proposed.

\begin{abstract}
Abbreviations
AUC: area under the curve; Cl: confidence interval; Cs: capillaries; DA: disease activity; dc: diffuse cutaneous; DLCO: diffusing capacity; ESR: erythrocyte sedimentation rate; ESSG: European Scleroderma Study Group; GC: giant capillaries; lc: limited cutaneous; MHE: micro-haemorrhages; mNEMO: modified NEMO score; mRSS: modified Rodnan skin score; MT: micro-thrombosis; NEMO: number of micro-haemorrhages; NVC: nailfold videocapillaroscopy; ROC: receiver operating characteristic; SSc: systemic sclerosis.
\end{abstract}

\section{Competing interests}

The authors declare that they have no competing interests. This study did not receive any funding support.

\section{Authors' contributions}

DS made substantial contributions to the conception and design of the work; acquisition, analysis, and interpretation of data; drafting the paper; and agreed to be accountable for all aspects of the work in ensuring that questions related to the accuracy or integrity of any part of the work are appropriately investigated and resolved. GS made substantial contribution to the design of the work; acquisition, analysis, and interpretation of data; revising the work critically for important intellectual content; and agreed to be accountable for all aspects of the work in ensuring that questions related to the accuracy or integrity of any part of the work are appropriately investigated and resolved. EZ contributed to acquisition of data; revising the work critically for important intellectual content; and agreed to be accountable for all aspects of the work in ensuring that questions related to the accuracy or integrity of any part of the work are appropriately investigated and resolved. WM contributed to the acquisition of data; revising the work critically for important intellectual content; and agreed to be accountable for all aspects of the work in ensuring that questions related to the accuracy or integrity of any part of the work are appropriately investigated and resolved. RP contributed to the interpretation of data; to revising the work critically for important intellectual content; and agreed to be accountable for all aspects of the work in ensuring that questions related to the accuracy or integrity of any part of the work are appropriately investigated and resolved. AMVA contributed to the interpretation of data; to revising the work critically for important intellectual content; and agreed to be accountable for all aspects of the work in ensuring that questions related to the accuracy or integrity of any part of the work are appropriately investigated and resolved. CV made substantial contribution to the design of the work; analysis and interpretation of data; drafting the work and revising it critically for important intellectual content; and agreed to be accountable for all aspects of the work in ensuring that questions related to the accuracy or integrity of any part of the work are appropriately investigated and resolved. NDP made substantial contributions to the design of the work; acquisition and interpretation of data; revising the work critically for important intellectual content; and agreed to be accountable for all aspects of the work in ensuring that questions related to the accuracy or integrity of any part of the work are appropriately investigated and resolved. All authors read and approved the final manuscript.

\section{Author details}

'U.O.C. Day Hospital Reumatologia, Ospedale Gaetano Pini, Via Gaetano Pini, 9-20122 Milano, Italy. ${ }^{2}$ Policlinico Universitario di Catania, Via Santa Sofia, 78, 95123 Catania, Italy. ${ }^{3}$ Policlinico Universitario Campus Bio-Medico di Roma, Via Álvaro del Portillo, 200, 00144 Rome, Italy. ${ }^{4}$ Istituto San Giuseppe, Via Alla Fonte, 7, 23900 Lecco, Italy.

Received: 15 May 2014 Accepted: 23 September 2014

Published online: 09 October 2014

\section{References}

1. LeRoy EC, Black C, Fleischmajer R, Jablonska S, Krieg T, Medsger TA Jr, Rowell N, Wollheim F: Scleroderma (systemic sclerosis): classification, subsets and pathogenesis. J Rheumatol 1988, 15:202-205.

2. Kahaleh B: The microvascular endothelium in scleroderma. Rheumatology (Oxford) 2008, 47:14-15.

3. Gabrielli A, Avvendimento EV, Krieg T: Scleroderma. N Eng J Med 2009, 360:1989-2003.

4. Del Papa N, Colombo G, Fracchiolla N, Mazzeo Moronetti L, Ingegnoli F, Maglione W, Comina DP, Vitali C, Fantini F, Cortellezzi A: Circulating endothelial cells as a marker of ongoing vascular disease in systemic sclerosis. Arthritis Rheumatol 2004, 50:1296-1304.

5. Nadashkevich O, Davis $P$, Fritzler M, Kovalenko W: A randomized unblinded trial of cyclophosphamide versus azathioprine in the treatment of systemic sclerosis. Clin Rheumatol 2006, 25:205-212.

6. Matucci-Cerinic M, Allanore Y, Czirják L, Tyndall A, Müller-Ladner U, Denton C, Valentini G, Distler O, Fligelstone K, Tyrrel-Kennedy A, Farge D, Kowal-Bielecka O, van den Hoogen F, Cutolo M, Sampaio-Barros PD, Nash P, Takehara K, Furst DE: The challenge of early systemic sclerosis for the EULAR Scleroderma Trial and Research group (EUSTAR) community. It is time to cut the Gordian knot and develop a prevention or rescue strategy. Ann Rheum Dis 2009, 68:1377-1380.

7. Stratton RJ, Wilson H, Black CM: Pilot study of anti-thymocyte globulin plus mycophenolate mofetil in recent-onset diffuse scleroderma. Rheumatology (Oxford) 2001, 40:84-88.

8. Valentini G, Della Rossa A, Bombardieri S, Bencivelli W, Silman AJ, D'Angelo S, Cerinic MM, Belch JF, Black CM, Bruhlman P, Czirjak L, De Luca A, Drosos AA, 
Ferri C, Gabrielli A, Giacomelli R, Hayem G, Inanc M, McHugh NJ, Nielsen H, Rosada M, Scorza R, Stork J, Sysa A, van den Hoogen FH, Vlachoyiannopoulos $P$ : European multicentre study to define disease activity criteria for systemic sclerosis. II. Identification of disease activity variables and development of preliminary activity indexes. Ann Rheum Dis 2001, 60:592-598.

9. Valentini G, Bencivelli W, Bombardieri S, D'Angelo S, Della Rossa A, Silman AJ, Black CM, Czirjak L, Nielsen H, Vlachoyiannopoulos PG: European multicentre study to define disease activity criteria for systemic sclerosis. III. Assessment of the construct validity of the preliminary activity criteria. Ann Rheum Dis 2003, 62:901-903.

10. Cutolo M, Pizzorni C, Secchi ME, Sulli A: Capillaroscopy. Best Pract Res Clin Rheumatol 2008, 22:1093-1108.

11. Van den Hoogen F, Khanna D, Fransen J, Johnson SR, Baron M, Tyndall A, Matucci-Cerinic M, Naden RP, Medsger TA Jr, Carreira PE, Riemekasten G, Clements PJ, Denton CP, Distler O, Allanore Y, Furst DE, Gabrielli A, Mayes MD, van Laar JM, Seibold JR, Czirjak L, Steen VD, Inanc M, Kowal-Bielecka O, Muller-Ladner U, Valentini G, Veale DJ, Vonk MC, Walker UA, Chung L, et al: 2013 Classification criteria for systemic sclerosis. An American College of Rheumatology/European League Against Rheumatism Collaborative Initiative. Arthritis Rheumatol 2013, 65:2737-2747.

12. Ingegnoli F, Boracchi $P$, Gualtierotti R, Lubatti C, Meani L, Zahalkova L, Zeni $S$, Fantini F: Prognostic model based on nailfold capillaroscopy for identifying Raynaud's phenomenon patients at high risk for the development of a scleroderma spectrum disorder. Arthritis Rheumatol 2008, 58:2174-2182.

13. Sulli A, Secchi ME, Pizzorni C, Cutolo M: Scoring the nailfold microvascular changes during the capillaroscopic analysis in systemic sclerosis patients. Ann Rheum Dis 2008, 67:885-887.

14. Corrado A, Carpagnano GE, Gaudio A, Foschino-Barbaro MP, Cantatore FP: Nailfold capillaroscopic findings in systemic sclerosis related lung fibrosis and in idiopathic lung fibrosis. Joint Bone Spine 2010, 77:570-574.

15. Cutolo M, Sulli A, Pizzorni C, Accardo S: Nailfold videocapillaroscopy assessment of microvascular damage in systemic sclerosis. J Rheumatol 2000, 27:155-160.

16. Wong ML, Highton J, Palmer DG: Sequential nailfold capillary microscopy in scleroderma and related disorders. Ann Rheum Dis 1988, 47:53-61.

17. Cutolo M, Sulli A, Smith V: How to perform and interpret capillaroscopy. Best Pract Res Clin Rheumatol 2013, 27:237-248.

18. Zufferey P, Depairon M, Chamot AM, Monti M: Prognostic significance of nailfold capillary microscopy in patients with Raynaud's phenomenon and scleroderma-pattern abnormalities. A six-year follow-up study. Clin Rheumatol 1992, 11:536-541.

19. Grassi W, Del Medico P: Microbleeding and capillary thrombosis. In Atlas of Capillaroscopy. Grassi and Del Medico: EDRA, Medical Publishing \& New Media, Milan; 2004:122-130.

20. Valentini $\mathrm{G}$ : The assessment of the patient with systemic sclerosis. Autoimmun Rev 2003, 2:370-376.

21. Clements PJ, Lachenbrunch PA, Seibold JR, Zee B, Steen VD, Brennan P, Silman AJ, Allegar N, Varga J, Massa M, Wigley FM, Ingenito F, Weisman M, White B, Martin RW, McCloskey D, Moreland LW, Mayes M, Lally EV, Unanue M, Cottier DH, Weiner S, Weinstein A, Medsger TA, Andrews B, Dixon M, Furst DE: Skin thickness score in systemic sclerosis: an assessment of interobserver variability in 3 independent studies. J Rheumatol 1993, 20:1892-1896.

22. Furst DE, Clements PJ, Steen VD, Medsger TA Jr, Masi AT, D'Angelo WA, Lachenbrunch PA, Grau RG, Seibold JR: The modified Rodnan skin score is an accurate reflection of skin biopsy thickness in systemic sclerosis. J Rheumatol 1998, 25:84-88.

23. Olski TM, Hunzelmann N, Krieg T: The skin in systemic scleroderma. In The Skin in Systemic Autoimmune Diseases, Volume 5. Edited by Sarzi-Puttini, Doria, Girolomoni, Kuhn. London: Elsevier; 2006:119-133.

24. De Angelis R, Grassi W, Cutolo M: A growing need for capillaroscopy in rheumatology. Arthritis Rheum 2009, 61:405-410.

25. Smith V, Decuman S, Sulli A, Bonroy C, Piettte Y, Deschepper E, de Keyser F, Cutolo M: Do worsening scleroderma capillaroscopic patterns predict future severe organ involvement? A pilot study. Ann Rheum Dis 2012, 71:1636-1639.

26. Sebastiani M, Manfredi A, Lo Monaco A, Praino E, Riccieri V, Grattagliano V, Bortoluzzi A, Stefanantoni K, D'Amico R, Giuggioli D, Ferri C: Capillaroscopic Skin Ulcers Risk Index (CSURI) calculated with different videocapillaroscopy devices: how its predictive values change. Clin Exp Rheumatol 2013, 31:115-117.
27. Tektonidou M, Kaskani E, Skopouli N, Moutsopoulos HM: Microvascular abnormalities in Sjögren's syndrome: nailfold capillaroscopy. Rheumatology (Oxford) 1999, 38:826-830.

28. Aslanidis S, Pyrpasopoulou A, Doumas M, Triantafyllou A, Chatzimichailidou S, Zamboulis C: Association of capillaroscopic microhaemorrages with clinical and immunological antiphospholipid syndrome. Clin Exp Rheumatol 2011, 29:307-309.

29. Bongard O, Bounameaux H, Miescher PA, De Moerloose P: Association of anticardiolipin antibodies and abnormal nailfold capillaroscopy in patients with systemic lupus erythematosus. Lupus 1995, 4:142-144.

30. Hosking SP, Bhatia R, Crock PA, Wright I, Squance ML, Reeves G: Non-invasive detection of the microvascular changes in a paediatric and adolescent population with type 1 diabetes: a pilot cross-sectional study. BMC Endocr Disord 2013, 13:41.

doi:10.1186/s13075-014-0462-8

Cite this article as: Sambataro et al:: Nailfold videocapillaroscopy micro-haemorrhage and giant capillary counting as an accurate approach for a steady state definition of disease activity in systemic sclerosis. Arthritis Research \& Therapy 2014 16:462.

\section{Submit your next manuscript to BioMed Central and take full advantage of:}

- Convenient online submission

- Thorough peer review

- No space constraints or color figure charges

- Immediate publication on acceptance

- Inclusion in PubMed, CAS, Scopus and Google Scholar

- Research which is freely available for redistribution

Submit your manuscript at www.biomedcentral.com/submit
C Biomed Central 